\title{
Dynamic properties of a reconstituted myelin sheath
}

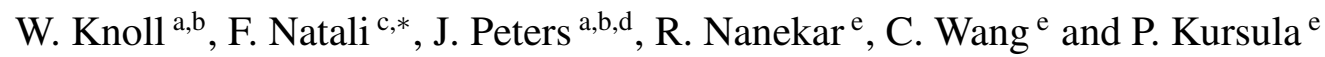 \\ ${ }^{a}$ University Joseph Fourier, Grenoble, France \\ ${ }^{\mathrm{b}}$ Institut Laue-Langevin, Grenoble, France \\ ${ }^{\mathrm{c}}$ CNR-IOM, OGG, c/o Institut Laue-Langevin, Grenoble, France \\ ${ }^{\mathrm{d}}$ Institut de Biologie Structurale, Grenoble, France \\ ${ }^{\mathrm{e}}$ Department of Biochemistry, University of Oulu, Oulu, Finland
}

\begin{abstract}
Myelin is a multilamellar membrane which, wrapping the nerve axons, increases the efficiency of nervous signal transmission. Indeed, the molecular components of the myelin sheath interact tightly with each other and molecules on the axonal surface to drive myelination, to keep both myelin and the axon intact, and to transduce signals from myelin to the axon and vice versa. Myelin is strongly affected in human demyelinating diseases in both the central and peripheral nervous system (CNS and PNS, respectively). Despite the presence of a well-defined set of myelin-specific proteins, little is known about the structure and the dynamics of these proteins, their interactions with the membrane and their influence on myelin stability. We present here the first neutron scattering results on the dynamics of the myelin sheath in PNS and of the interaction between its constituents. Specifically, the human P2 protein is shown to stabilize the lipid membrane upon binding to it.
\end{abstract}

Keywords: Membrane dynamics, neutron scattering, myelin

\section{Introduction}

Biological membranes serve diverse basic functions in eukaryotic and prokaryotic cells, regulating the exchange of matter, energy and information with the surrounding environment. In a functional sense, proteins obviously play a pertinent role in many of these functions. Yet, their lateral organization within membranes is often highly dynamic, causing their transient localization in different lipid environments that may affect protein structure, their oligomerization and, thereby, function.

Myelin is the lipid-rich, multilamellar membrane responsible for the speed-up of the nerve impulse conduction in vertebrates (up to $100 \mathrm{~m} / \mathrm{s}$ in a healthy person). Myelin is destroyed by autoimmune processes in human demyelinating diseases, including multiple sclerosis (MS) in CNS and peripheral neuropathies (PN) in PNS. MS is characterized by patches of demyelination [10] that can be scattered throughout the white matter of the CNS, but are usually localized in the brain stem, periventricular areas, the optic nerve and the cervical spinal cord. These multiple scars (or scleroses) cause symptoms specific to the interrupted signals.

The biochemical composition of myelin includes only a few major proteins [19]; two proteins, the proteolipid protein (PLP) and the myelin basic protein (MBP) account for $80 \%$ of the total CNS myelin protein. CNS and PNS myelin differ in their protein constituents. MBP is also an abundant component

\footnotetext{
${ }^{*}$ Corresponding author: Dr. F. Natali, CNR-IOM, c/o Institut Laue-Langevin, BP 1566, rue Jules Horowitz, 38042 Grenoble Cedex 9, France. Tel.: +33 4762070 71; Fax: +33 4762076 88; E-mail: natali@ill.fr.
} 
of PNS myelin [11], where it colocalizes with another myelin-specific protein, P2. The P2 protein, while missing in CNS, represents $1-15 \%$ of the total myelin protein in the PNS.

MBP is essential for the formation of CNS myelin, able to interact with a wide range of ligands, often polyanionic in nature [2]. Due to its high charge and low overall hydrophobicity, there is significant intramolecular electrostatic repulsion in MBP; this results in MBP being an extended, intrinsically unstructured protein in solution [12,21].

$\mathrm{P} 2$ is a small folded protein with a high homology to members of the fatty acid binding protein family [7,18]. It is expressed in the cytoplasmic face of compact myelin in the PNS [9,27], and its lipid-binding activity suggests it may serve important functions in generating and maintaining the unique lipid composition of myelin [28]. P2 is possibly one of the autoantigens in the human disorder Guillain-Barre syndrome [14,15], an autoimmune disease of the PNS.

Although the main subject of myelin research often concerns its proteins, dynamic changes in proteinlipid interactions and lipid-based membrane microdomains also have a role in the etiology of MS and PN. Detailed knowledge of the fine structure and dynamics of myelin at the level of lateral membrane domain composition, and lipid-protein interactions within such domains, is imperative for understanding the unknown pathogenic mechanisms of demyelination.

Biochemical and structural studies on myelin flourished during the 1970s and 1980s [3-6,16,26,29], and correlations have been observed between myelin structural modifications [13,22] and some common neurological degenerative events of pathological nature in humans. In this context, wide angle X-ray diffraction studies [5] revealed that the lipid phase transition temperature of MS myelin is $20^{\circ} \mathrm{C}$ lower than that of normal myelin, indicating differences in the structural organization of the bilayer.

Dynamic properties are of great importance at the molecular level of biology and the marked temperature dependence of the activity of biomolecules reflects their thermal mobility. Dynamic events in biomolecular systems occur on a very large time-scale, ranging from femtoseconds to nearly seconds. Within this broad interval, motions occurring in the pico- to nano-second time-scale are of particular interest and relevance, since they cover the transition region from 'discrete' local excitations of small molecular subunits to slower processes involving collective motions of massive parts of macromolecular assemblies. This time window is well covered by inelastic and quasielastic neutron scattering, These techniques can, therefore, play a relevant role in improving the understanding of molecular motions which affect the functionality of biomolecules.

We have demonstrated, during the last five years [8,23-25], the power of the neutron scattering technique as an optimal probe for the dynamics of the myelin sheath and its components. Our studies so far focused on the effect of MBP on the global membrane dynamics of ordered dimyristoyl L-a-phosphatidic acid (DMPA) assemblies, simulating the myelin sheath in the CNS. The study covered the lipid gel (phase $\alpha$ ) to liquid (phase $\beta$ ) phase transition $\left(T_{\alpha \rightarrow \beta}=320 \mathrm{~K}\right.$ ). Strong anisotropy in the dynamic behaviour depended on the membrane composition, and MBP was found to strongly enhance the out-ofplane mobility of the lipid molecules in the liquid phase. In this study, we now address the PNS system, and effects of both MBP and P2 on liposome membranes are investigated.

\section{Materials and methods}

\subsection{Protein purification}

Details of the protein purification protocols will be published elsewhere. In brief, His-tagged human P2 was expressed in E. coli and purified using immobilized metal ion affinity chromatography. The His- 
tag was cleaved off with recombinant TEV protease and purification was completed by gel filtration. The recombinant His-tagged $\mathrm{C} 1$ and $\mathrm{C} 8$ isoforms of murine $18.5 \mathrm{kDa}$ MBP were expressed in $E$. coli and purified by slight modifications of previously published procedures [1]. All protein samples were lyophilized prior to shipment to the neutron source and final sample preparation.

\subsection{Sample preparation and characterization}

1,2-dimyristoyl-sn-glycero-3-phosphate (DMPA), 1,2-dioleoyl-sn-glycero-3-phosphocholine (DOPC) and 1,2-dioleoyl-sn-glycero-3-phospho-L-serine (DOPS) were purchased as dry powders from Lipoid Company (Germany). After dissolving the lipids in chloroform and subsequent drying with nitrogen gas, they were solubilised in $\mathrm{D}_{2} \mathrm{O}$. By extruding the lipid solution through a $100 \mathrm{~nm}$ pore sized membrane, using the Avanti ${ }^{\circledR}$ Mini-Extruder, unilamellar vesicles were prepared. To compare liposomes of DMPA and DOPS + DOPC with these liposomes mixed with the MBP or P2 proteins, the proteins were added to one part of the lipid solution before the extrusion. The hydrodynamic radius $\left(R_{\mathrm{h}}\right)$ of the liposomes, with and without proteins, was estimated by dynamic light scattering (DLS). The experiments were carried out at the EMBL outstation in Grenoble using the ALV CGS-3 Compact Goniometer. The obtained correlation intensities were normalized to the measurement of a standard for which toluene is typically used. The suitable lipid concentration for DLS measurement was found to be of $0.001 \mathrm{mg} / \mathrm{ml}$. Dust particles were removed by using a filter with $20 \mu \mathrm{m}$ pores.

\subsection{Neutron scattering experiment}

Incoherent elastic neutron scattering measurements as a function of temperature were performed on the thermal $(\lambda=2.23 \AA$ ) high-energy resolution backscattering spectrometer IN13 (Institut LaueLangevin, Grenoble, France), characterized by a very large momentum transfer range $(0.2<Q<$ $4.9 \AA^{-1}$ ) with a good and nearly $Q$-independent energy resolution ( $8 \mu \mathrm{eV}$ FWHM). IN13, therefore, allows accessing the space and time windows of $1-6 \AA$ and $0.1 \mathrm{~ns}$ respectively.

The elastic scattering intensities $\left(I_{\mathrm{el}}(Q)=S(Q, \omega=0)\right)$, properly corrected for the empty sample holder signal, were normalized with respect to a vanadium scan (typically used as a standard), to compensate for spurious background contributions and detector efficiency. The sample mass and thickness were suitably chosen to optimize the compromise between good signal-to-noise ratio and multiple scattering contributions. For this purpose, a transmission of approximately $90 \%$ was guaranteed using $100 \mathrm{mg}$ of powder per sample allocated in $1.5 \mathrm{~mm}$ thick aluminum sample holders.

\section{Results and discussion}

The temperature region ( $278<T<313 \mathrm{~K}$ ) was chosen in order to (1) avoid any Bragg reflections arising from ice assigned to free water molecules in- and outside the liposome ( $T>273 \mathrm{~K})$ and (2) prevent $\mathrm{P} 2$ from denaturation $(T<320 \mathrm{~K})$. In order to investigate how the membrane dynamics are affected by the presence of $\mathrm{P} 2$ in both the gel and liquid lipidic phase (which would allow us a direct comparison to our previous study in CNS), two different liposome compositions were chosen. The DMPA artificial membrane shows a lipid $L_{\alpha} \rightarrow L_{\beta}$ phase transition at $T=320 \mathrm{~K}$. Thus, in the whole $273<T<315 \mathrm{~K}$ region, DMPA is in the gel phase. On the other hand, two-component liposomes, made of a mixture of neutral (DOPC) and charged (DOPS) lipids, at 1:1 w/w ratio, were chosen to investigate the liquid 
lipid phase. Indeed, both DOPC and DOPS show a lipid $L_{\alpha} \rightarrow L_{\beta}$ phase transition well below room temperature (DOPC: $T_{\alpha \rightarrow \beta}=253 \mathrm{~K}$; DOPS: $T_{\alpha \rightarrow \beta}=264 \mathrm{~K}$ ). Thus, the mixed lipid compound is in the liquid phase in the entire $T$ range investigated here.

While DMPA was chosen to compare results in PNS to our previous investigation in CNS [23-25], the neutral-charged mixture was chosen to better represent the lipid composition in myelin. The chosen P2 concentration ( $1.25 \%$ of the total sample mass) allows neglecting the scattering arising from the protein (5\% at maximum of the total scattering signal). Thus, any differences in the neutron response can be unambiguously assigned to changes in membrane dynamics induced by the presence of the P2 protein.

In Fig. 1, we report the incoherent elastic intensity of DMPA liposomes vs. the square of momentum transfer. The fast decrease of the intensity observed for all the temperatures investigated is a clear sign of enhanced dynamics that may count also for some additional quasi-elastic components to the elastic scattering. In particular, two main effects may contribute to the QENS additional terms: (1) confined water inside the liposomes, and (2) liposome diffusion in the heavy water medium. $\mathrm{D}_{2} \mathrm{O}$ was chosen as solvent, indeed, to minimize the first contribution to the total scattering intensity. However, while free water outside the liposomes does not participate in our signal since it counts as flat background in the energy window explored here, the heavy water molecules inside the liposomes may include at least two different populations: free (at the center of the liposomes) and bound (closer to the lipid polar heads of the internal leaflet) molecules. The latter may give rise to a quasi-elastic contribution, which can fall within the energy resolution of the experiment, thus affecting the correct interpretation of the elastic data, since it results in a spurious decrease of the elastic intensity. Thus, bound water molecules have to be taken into account for a correct data interpretation. Also liposome diffusion has to be properly

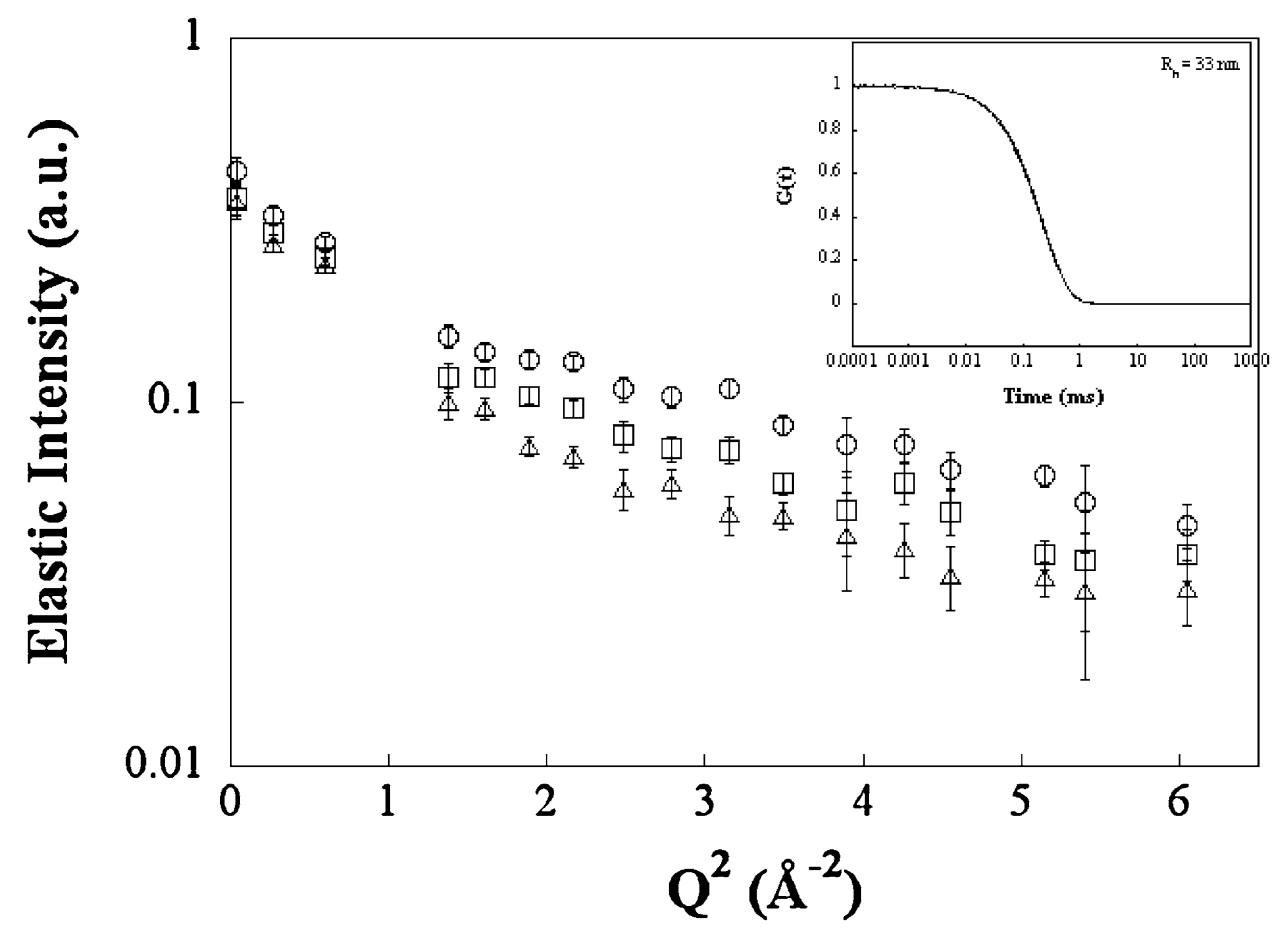

Fig. 1. Normalized elastic intensity of DMPA liposomes vs. T. For clarity, only 3 temperatures are shown (circles: 277 K; squares: $287 \mathrm{~K}$; triangles: $297 \mathrm{~K}$ ). Insert: experimental DLS correlation functions of DMPA liposomes measured before and after the neutron experiments (continuous and dotted lines, respectively). 
considered, since at the used lipid concentration $(50 \mathrm{mg} / \mathrm{ml})$, the system has to be considered as crowded, thus speeding down the lipid diffusion constant to values observable on IN13. To identify and quantify all the different contributions to our elastic data, further QENS experiments are planned on several complementary instruments, allowing us to span a wide energy range $(1-100 \mu \mathrm{eV})$.

In order to check the quality of the sample we systematically performed DLS measurements before and after the neutron experiments. In the insert of Fig. 1, we report, as an example, the experimental time dependent correlation function $G(t)$ of DMPA liposomes measured before and after the neutron experiments. The two curves overlap perfectly and can be fitted using a single and narrow liposome population of hydrodynamic radius $R_{\mathrm{h}}=33 \mathrm{~nm}$ and a very low polidispersity $(p=0.98)$.

The proper protein-liposome binding is currently checked by gel electrophoresis using a sucrose gradient protocol [17].

The influence of the composition of the two protein-free liposome samples used in this work (DMPA and DOPC + DOPS) is shown in Fig. 2, where the integrated elastic intensities are reported as a function of the explored $T$ range. In order to gain in statistics, data have been binned over the interesting $Q$ region where the non-zero intensities are still appreciable $\left(Q^{2} \leqslant 6 \AA^{-2}\right)$. A remarkable difference is observed, supporting a damping of membrane dynamics in the DMPA system. This may be assigned to the different lipid phases explored by the two types of liposomes in the measured $T$ region. As expected, indeed, the liquid phase at which DOPC + DOPS liposomes are trapped reflects much higher lipid motion. However, a contribution from a different behavior of the dynamics of the confined water molecules inside the liposomes cannot be excluded, due to a difference in the charge distribution on the lipid inner surface; the neutral DOPC lipids behave as spacers, probably causing a change in the lipid polar head-water molecules interactions.

The mean square displacements (MSD) extracted for the protein-free and P2-bound liposomes are reported in Fig. 3. All data on MBP bound liposomes are here only briefly commented and not shown, even

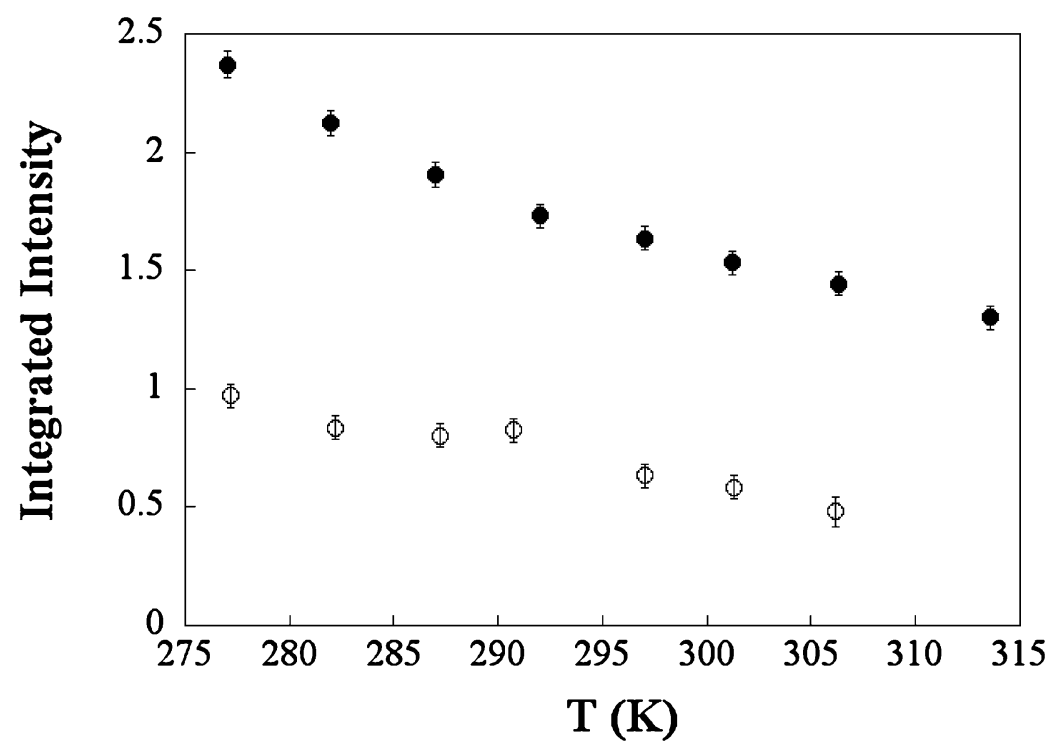

Fig. 2. Comparison of temperature dependence of the normalized elastic intensity of mono and bi-component liposomes. To gain in statistics, data are integrated over the $Q^{2}$ region up to $6 \AA^{-2}$ (DMPA: filled symbols; DOPC + DOPS: empty symbols). 


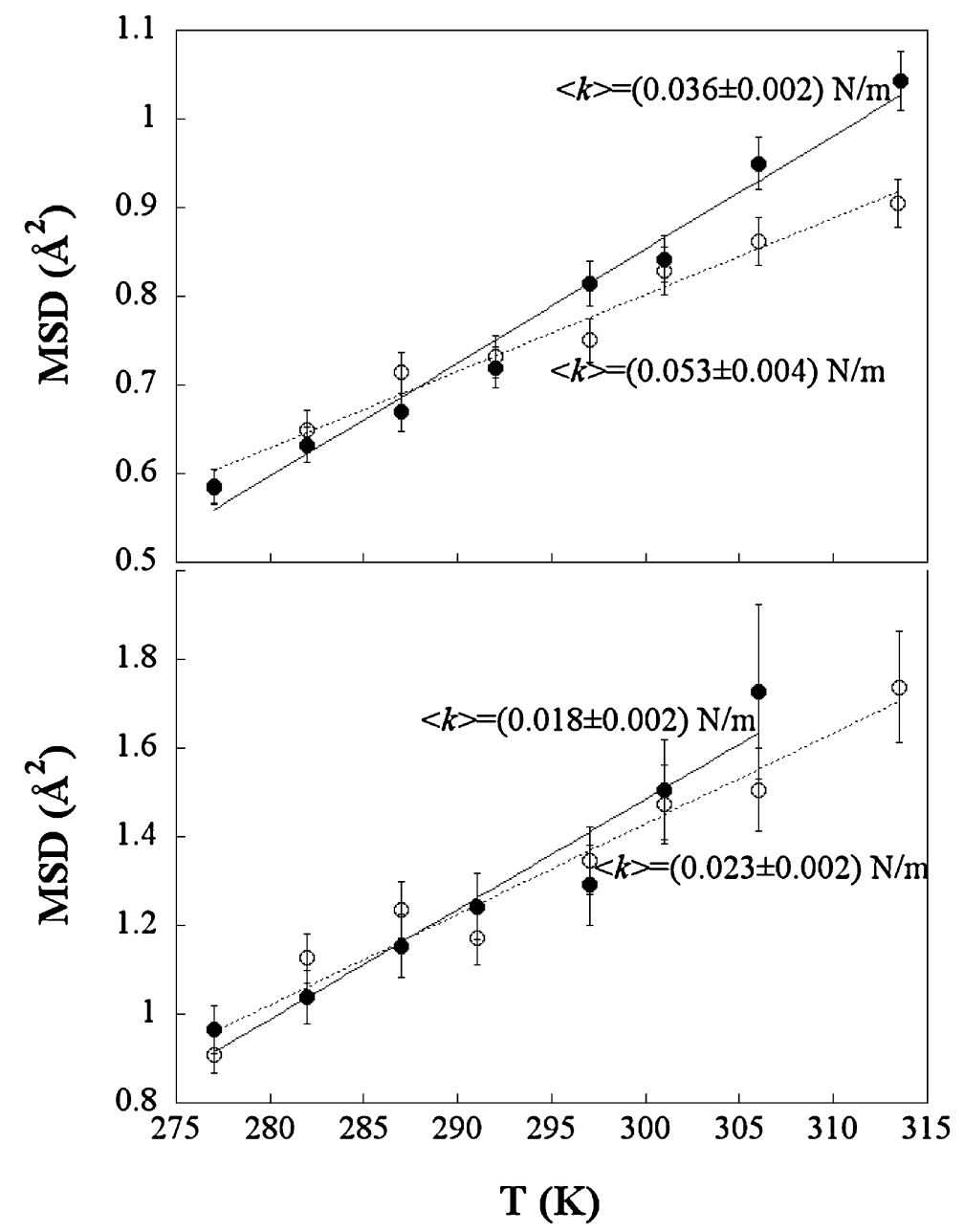

Fig. 3. Temperature dependence of the mean square displacements of proteolipid complexes compared to their respective liposomes. Upper panel: DMPA complexes; lower panel: DOPC + DOPS complexes. Empty symbols: liposomes with P2; filled circles: protein-free liposomes. The $k$ force constants, estimated using the force constant model, are also reported.

though in perfect agreement to previous results [23-25], because of the poor sample quality suggested by significant changes in the DLS spectra as a function of time. The MSD values have been calculated from the slope of the elastic intensity vs. $Q^{2}$, in the low $Q$ limit, where the Gaussian approximation is valid. The MSD count for both harmonic and anharmonic contributions of the all proton dynamics. A detailed description of the MSD extraction protocol may be found elsewhere [20]. The binding of P2 to the liposomes shows the same effect on the two lipocomplexes studied here: $\mathrm{P} 2$ is found to enhance the membrane stability, reducing the lipid dynamics. Using the free constant model successfully introduced by Zaccai in 2000 [30], the effective force constants ( $k$ parameters) have been estimated for our compounds (Fig. 3). The $k$ constants are an indirect measure of the stability of a system; higher $k$ values refer to a more rigid system, confirming the property of $\mathrm{P} 2$ to enhance the membrane stability, which may play a key role in proper nerve impulse transmission. 


\section{Conclusions}

P2 is a highly abundant protein of the PNS myelin membrane, with putative roles in the formation and homeostasis of myelin; the relationships between the structure, function, and dynamics of P2 have, however, been poorly characterized. The results presented here strongly suggest a stabilizing effect induced by the $\mathrm{P} 2$ protein on the membrane dynamics, independently from the liposome composition (in particular from the net charge density of the liposomes) and from the lipidic phase transition in which the liposomes are trapped (gel phase in DMPA and liquid phase in DOPC + DOPS). One possible physical explanation for the observed higher rigidity may account for the recent observation (data not yet published) by molecular dynamics simulation, of the partial disposition of the P2 protein inside the lipid bilayer and on its surface, affecting in such a way both in plane and out-of-plane lipid motions.

In the near future, QENS experiments are planned to get better insight into the properties exerted by $\mathrm{P} 2$, in particular to map selectively the different lipid motions, which may be affected by the lipid-protein interaction. Complementary elastic experiments will also soon be performed on the same liposome compositions, where the free water molecules will be removed by promoting oriented multibilayer formation, followed by drying treatment, in order to extend the study of the DOPC + DOPS liposomes below room temperature, i.e., in the gel phase. This will allow us to discriminate between net charge density, lipid phase and chemical lipid composition contributions. Taken together, our results elucidate the functional properties of a quantitatively major component of vertebrate PNS myelin.

\section{Acknowledgements}

We would like to express our gratitude to Mrs. Iulia Blesneac, Ms. Niina Torniainen and Ms. Viivi Majava for their help in sample preparation and characterization. This work has been supported by the Academy of Finland and the Sigrid Juselius Foundation (Finland).

\section{References}

[1] I.R. Bates, P. Matharu, N. Ishiyama, D. Rochon, D.D. Wood, E. Polverini, M.A. Moscarello, N.J. Viner and G. Harauz, Characterization of a recombinant murine 18.5-kDa myelin basic protein, Protein Expr. Purif. 20 (2000), 285-299.

[2] J.M. Boggs, Myelin basic protein: a multifunctional protein, Cell. Mol. Life Sci. 63 (2006), 1945-1961.

[3] C. Brunner, H. Lassmann, T.V. Waehneldt, J.M. Matthieu and C. Linington, Differential ultrastructural localization of myelin basic protein, myelin/oligodendroglial glycoprotein, and $2^{\prime}, 3^{\prime}$-cyclic nucleotide $3^{\prime}$-phosphodiesterase in the CNS of adult rats, J. Neurochem. 52 (1989), 296-304.

[4] D.L. Caspar and D.A. Kirschner, Myelin membrane structure at 10 Å resolution, Nat. New Biol. 19 (1971), 46-52.

[5] L.S. Chia, J.E. Thompson and M.A. Moscarello, Alteration of lipid-phase behavior in multiple sclerosis myelin revealed by wide-angle X-ray diffraction, Proc. Natl. Acad. Sci. USA 81 (1984), 1871-1874.

[6] L.S. Chia, J.E. Thompson and M.A. Moscarello, X-ray diffraction evidence for myelin disorder in brain from humans with Alzheimer's disease, Biochim. Biophys. Acta 775 (1984), 308-331.

[7] A. Chmurzynska, The multigene family of fatty acid-binding proteins (FABPs): function, structure and polymorphism, J. Appl. Genet. 47 (2006), 39-48.

[8] A. Deriu and F. Natali, Recent neutron investigation on biomolecular dynamics: from model systems towards complex macromolecular machines, J. Neutron Research 10 (2002), 115-122.

[9] E.H. Eylar, I. Szymanska, A. Ishaque, J. Ramwani and S. Dubiski, Localization of the P2 protein in peripheral nerve myelin, J. Immunol. 124 (1980), 1086-1092.

[10] R.J. Franklin, Why does remyelination fail in multiple sclerosis?, Nat. Rev. Neurosci. 3 (2002), 705-771.

[11] B. Garbay, A.M. Heape, F. Sargueil and C. Cassagne, Myelin synthesis in the peripheral nervous system, Prog. Neurobiol. 61 (2000), 267-304. 
[12] G. Harauz, N. Ishiyama, C.M. Hill, I.R. Bates, D.S. Libich and C. Farès, Myelin basic protein-diverse conformational states of an intrinsically unstructured protein and its roles in myelin assembly and multiple sclerosis, Micron 35 (2004), 503-542.

[13] A. Hirano, S.D. Cook, J.N. Whitaker, P.C. Dowling and M.R. Murray, Fine structural aspects of demyelination in vitro. The effects of Guillain-Barré serum, J. Neuropathol. Exp. Neurol. 30 (1971), 249-265.

[14] R.A. Hughes and D.R. Cornblath, Guillain-Barre syndrome, Lancet 366 (2005), 1653-1666.

[15] R.A. Hughes, R.D. Hadden, N.A. Gregson and K.J. Smith, Pathogenesis of Guillain-Barre syndrome, J. Neuroimmunol. 100 (1999), 74-97.

[16] D.A. Kirschner and A.L. Ganser, Compact myelin exists in the absence of basic protein in the shiverer mutant mouse, Nature 283 (1980), 207-210.

[17] J. Knol, Membrane reconstitution and functional analysis of a sugar transport system, PhD dissertation, University of Groningen, Groningen, 1999.

[18] P. Kursula, Structural properties of proteins specific to the myelin sheath, Amino Acids 34 (2008), 175-185.

[19] C. Laule, I.M. Vavasour, S.H. Kolind, D.K. Li, T.L. Traboulsee, G.R. Moore and A.L. MacKay, Magnetic resonance imaging of myelin, Neurotherapeutics 4 (2007), 460-484.

[20] U. Lehnert, V. Reat, M. Weik, G. Zaccaï and C. Pfister, Thermal motions in bacteriorhodopsin at different hydration levels studied by neutron scattering: correlation with kinetics and light-induced conformational changes, Biophys. J. 75 (1998), $1945-1952$.

[21] V. Majava, C. Wang, M. Myllykoski, S.M. Kangas, S.U. Kang, N. Hayashi, P. Baumgärtel, A.M. Heape, G. Lubec and P. Kursula, Structural analysis of the complex between calmodulin and full-length myelin basic protein, an intrinsically disordered molecule, Amino Acids 39 (2010), 59-71.

[22] E.B. Masurovsky, M.B. Bunge and R.P. Bunge, Cytological studies of organotypic cultures of rat dorsal root ganglia following X-irradiation in vitro. II. Changes in Schwann cells, myelin sheaths, and nerve fibers, J. Cell Biol. 32 (1967), 497-518.

[23] F. Natali, A. Gliozzi, R. Rolandi, P. Cavatorta and A. Deriu, Myelin basic protein reduces molecular motions in DMPA, an elastic neutron scattering study, Appl. Phys. A 74 (2002), 1582-1584.

[24] F. Natali, A. Relini, A. Gliozzi, R. Rolandi, P. Cavatorta, A. Deriu, A. Fasano and P. Riccio, Protein-membrane interaction: effect of myelin basic protein on the dynamics of oriented lipids, Chem. Phys. 292 (2003), 455-464.

[25] F. Natali, A. Relini, A. Gliozzi, R. Rolandi, P. Cavatorta, A. Deriu, A. Fasano and P. Riccio, The influence of the lipidprotein interaction on the membrane dynamics, Physica B 350 (2004), E623-E626.

[26] P. Riccio, A. Tsugita, A. Bobba, F. Vilbois and E. Quagliariello, A new protein of the brain myelin: isolation and chemical characterization, Biochem. Biophys. Res. Commun. 127 (1985), 484-492.

[27] B.D. Trapp, M. Dubois-Dalcq and R.H. Quarles, Ultrastructural localization of P2 protein in actively myelinating rat Schwann cells, J. Neurochem. 43 (1984), 944-948.

[28] K. Uyemura, K. Yoshimura, M. Suzuki and K. Kitamura, Lipid binding activities of the P2 protein in peripheral nerve myelin, Neurochem. Res. 9 (1984), 1509-1514.

[29] C.R. Worthington and T.J. McIntosh, Direct determination of the lamellar structure of peripheral nerve myelin at moderate resolution (7A), Biophys. J. 14 (1974), 703-729.

[30] G. Zaccai, How soft is a protein? A protein dynamics force constant measured by neutron scattering, Science $\mathbf{2 8 8}$ (2000), 1604-1607. 


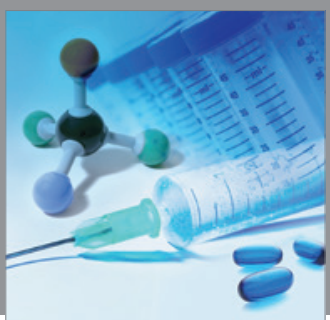

International Journal of

Medicinal Chemistry

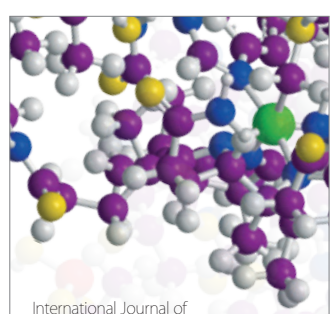

Carbohydrate Chemistry

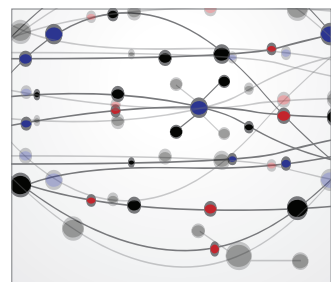

The Scientific World Journal
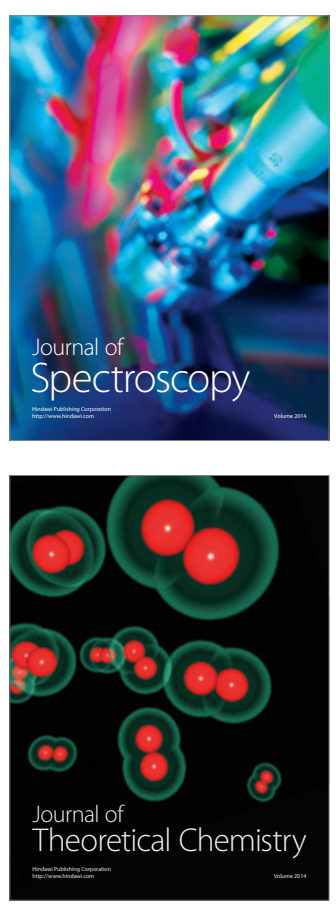
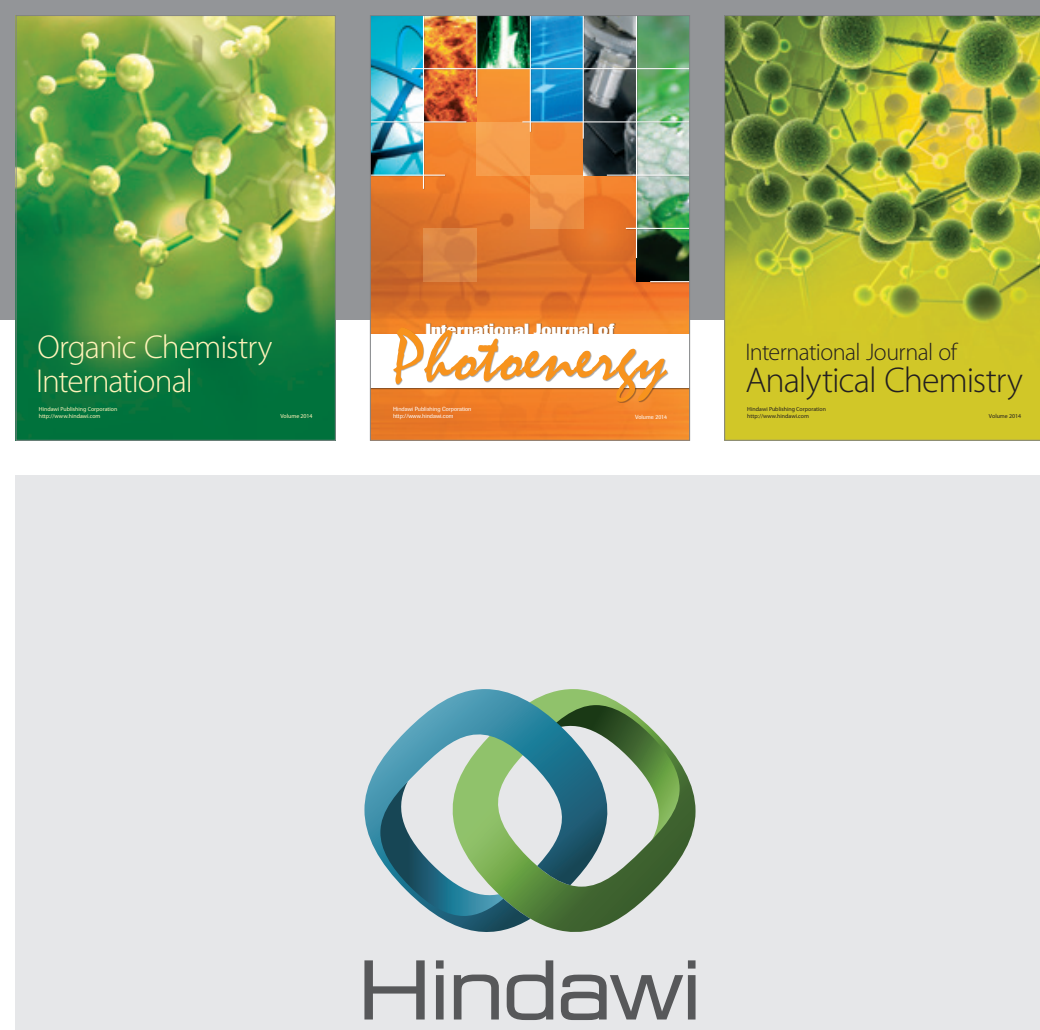

Submit your manuscripts at

http://www.hindawi.com
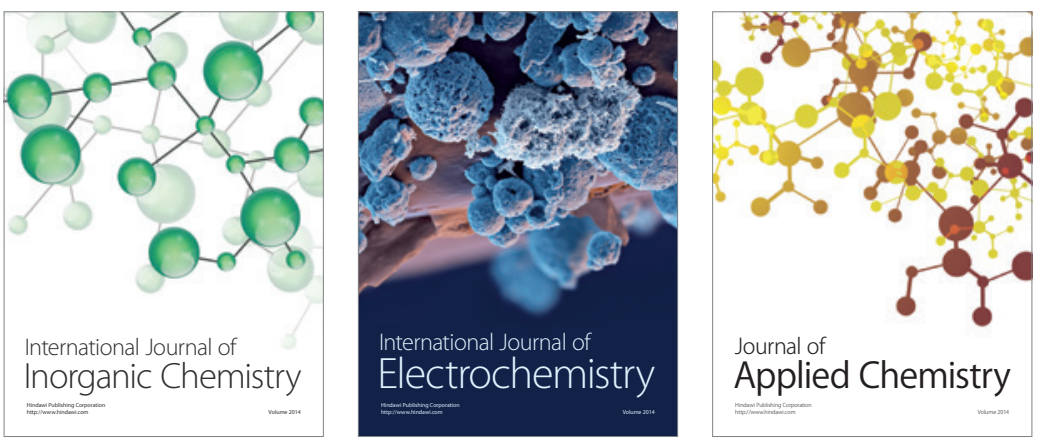

Journal of

Applied Chemistry
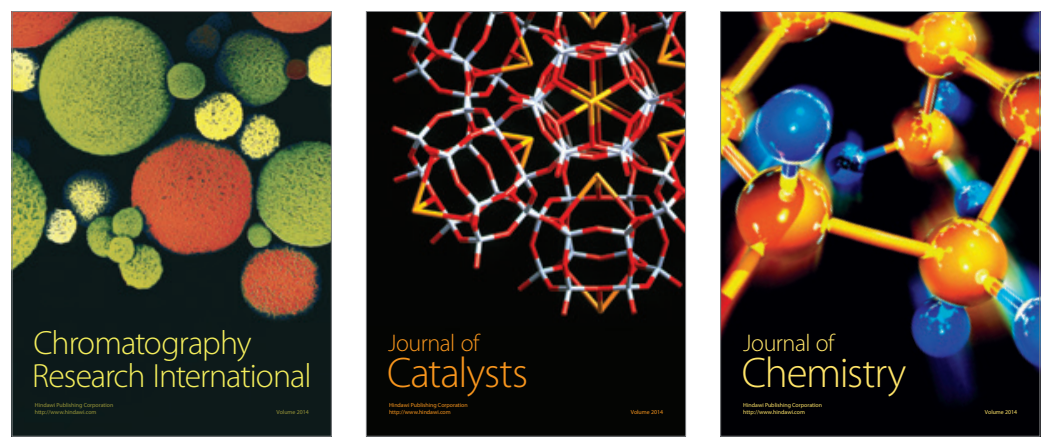
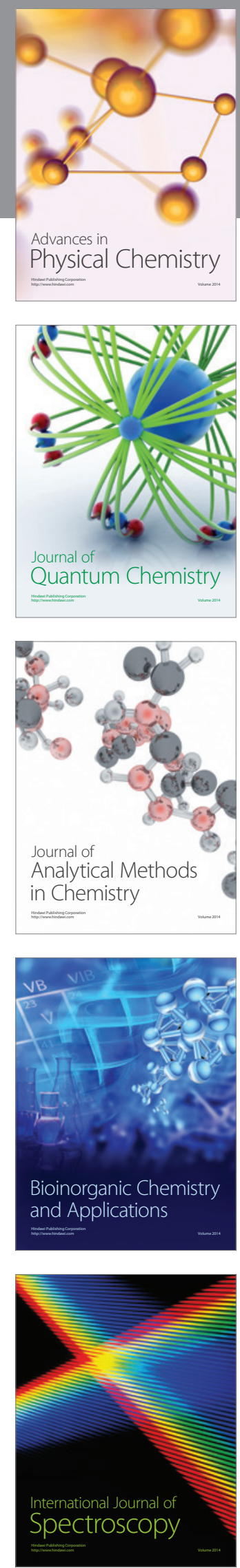